\title{
Synthesis of a Linear Antenna Array for Maximum Side- lobe Level Reduction
}

\author{
S.A. Babale \\ Department of Electrical and \\ Computer Engineering, \\ Bayero University, Kano, \\ Nigeria
}

\author{
D.D. Dajab \\ Departments of and \\ Computer Engineering, \\ Ahmadu Bello University, \\ Zaria, Nigeria
}

\author{
K. Ahmad \\ Departments of and Computer \\ Engineering, \\ Ahmadu Bello University, \\ Zaria, Nigeria
}

\begin{abstract}
There are number of Techniques used to reduce Side Lobe Level (SLL) of antenna arrays to save power and improve Quality of Service (QoS) by ensuring maximum radiation in desired direction, several methods are available in literature. It is a well known fact that when an attempt is made to reduce the first sidelobe level of an antenna array, the beamwidth increases. However, in the present work, an array for a specified first side lobe level of $-35 \mathrm{~dB}$ was synthesized using Dolph Chebyshev method. A real-value Genetic Algorithm (RGA) was also used to optimize the current excitations. The weighting vectors are compared. Using these vectors, patterns are generated for arrays of different elements. The resultant patterns are compared and the result shows greater improvement in the SLL reduction from the RGA method without deteriorating the main beamwidth.
\end{abstract}

\section{Keywords}

Side lobe level, Quality of Service, beamwidth, Dolph Chebyshev, and Real-Value Genetic Algorithm

\section{INTRODUCTION}

An antenna array is a group of identical antenna elements arranged usually in a regular fashion. In a linear array, the elements are arranged along a straight line and are often fed coherently. The currents through the elements are generally different in amplitudes and phases in order to obtain greater control over the radiation pattern [1]. Basically the antenna array design involves calculating the complex currents of the individual antenna elements and selecting an appropriate antenna element. The current excitations would largely determine how sharp the resultant radiation pattern is and how small the sidelobe levels are in comparison to the main lobe [2-5].

In most of the applications, side lobes are generally unwanted and the first side lobe level usually introduces Electromagnetic Interference (EMI) problems and hence it should be lowered without disturbing the beam width [6, 7]. Side lobe level (SLL) reduction has a great importance in recent communication systems. It is considered as one of the most important applications of digital beamforming since it reduces the effect of interference arriving outside the main lobe. This interference reduction increases the capacity of the communication systems [8].

In some applications such as radar system, SLL suppression is one of a critical topic of research. It plays an important role in anti-jamming technique. Genetic Algorithm (GA) is an efficient method with parallel, random and global search and optimization. It is applied widely in array processing, as well as in the analysis and design of patterns. Recently, a GA optimization is used to reduce the sidelobe level for linear array antennas as proposed in $[2,4,5,8,9]$. It is evident that when the first side lobe is reduced, null to null beamwidth is increases. This leads to great disturbance in the directivity of the array. In this paper, two methods were used for the synthesis of the antenna array. They are Dolph Chebyshev and RGA. With Dolph Chebyshev method, the excitation coefficients are obtained and the patterns are presented for different arrays. In order to reduce the first sidelobe further, RGA is applied to find out other sets of excitation coefficients. The resultant patterns of RGA are presented and compared with those of Dolph Chebyshev.

\section{ANTENNA ARRAY SYNTHESIS}

In antenna design, it is required to obtain narrowest main beam width along with low side lobe levels. It was found that as the current amplitude taper from the center to the edges of the array increased, the side-lobe level decreased, but with an increase in the width of the main beam. In most applications, it is desirable to have both a narrow main beam as well as low side lobes. It would, therefore be useful to have a pattern with an optimum compromise between beamwidth and side-lobe level [7].

As might be expected, optimum beamwidth-side-Iobe level performance occurs when there are as many side lobes in the visible region as possible and they have the same level. Dolph recognized that Chebyshev polynomials possess this property, and he applied them to the synthesis problem. Chebyshev polynomial is defined by

$$
T_{n}(x)=\left\{\begin{array}{lr}
(-1)^{n} \cosh \left(n \cosh ^{-1}|x|,\right. & x<-1 \\
\cos \left(n \cos ^{-1} x,\right. & -1<x<1 \\
\cosh \left(n \cosh ^{-1} x,\right. & x>1
\end{array}\right.
$$

The recursive formula given below can be used to obtain higher order polynomials:

$$
T_{n+1}(x)=2 x T_{n}(x)-T_{n-1}(x)
$$

For example, $T_{0}(x), T_{1}(x)$, and $T_{2}(x)$ are:

$$
\begin{aligned}
& T_{0}(x)=1 \\
& T_{0}(x)=x \\
& T_{2}(x)=12 x^{2}-1
\end{aligned}
$$

\section{GENETIC ALGORITHMS}

Genetic Algorithms are a family of computational models inspired by evolution. GA can be used to find approximate solutions to search problems through the application of the principles of evolutionary biology. GA uses biologically inspired techniques, such as genetic inheritance, natural selection, mutation, and sexual reproduction (crossover or recombination) [10]. The method was developed by John Holland in 1975. The method is used for solving both 
constrained and unconstrained optimization problems that is based on natural selection, the process that drives biological evolution. The algorithm repeatedly modifies a population of individual solutions. At every step, an individual is selected at random from the current population to form the parents and uses them to produce the children (offspring) for the next generation. Over successive generations, the population "evolves" toward an optimal solution. GA's can be used when fitness function is discontinuous, non differentiable, stochastic, or highly nonlinear [5].The flowchart of the reproduction cycle is shown in Figure 1. The algorithm is summarized in the following steps:

1. Create an initial population by randomly generating an initial population within the variable constraint range.

2. Evaluate and saves the fitness for each individual in the current population based on the objective function.

3. Define the selection probability for each individual so that it is proportional to its fitness.

4. Generates the next population by probabilistically selecting the individuals from the previous current population to produce offspring via genetic operators.

5. Terminate run after maximum number of generation are attained or repeats step 2 until a satisfactory solution is obtained.

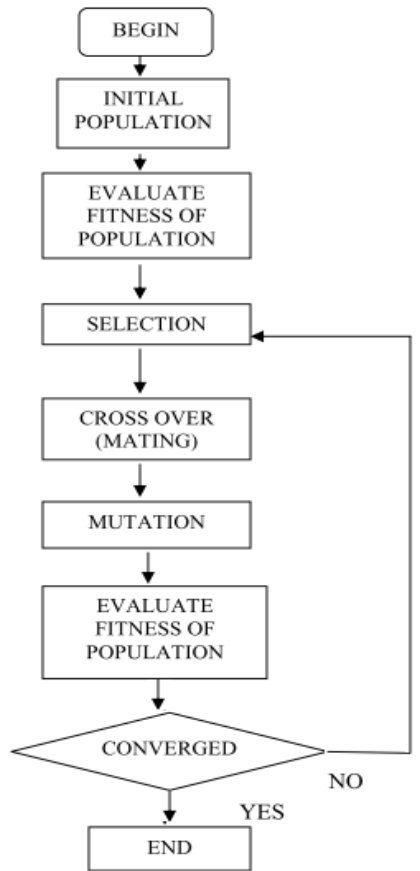

Figure 1: Genetic algorithm reproduction cycle [5].

\section{DESIGN EQUATIONS}

The simplest array geometry is known to be a linear array. It is an array whose elements are aligned along a straight line and generally have a uniform inter element spacing. Linear arrays are the simplest to analyze and many valuable insights can be gained by understanding their behavior [11]. An Nelement linear array shown in Figure 2 composed of isotropic radiating antenna elements. It is assumed that all the antenna elements are symmetric about the center of the linear array. The far field array factor of this array with an even number of isotropic elements, $2 \mathrm{~N}$ can be expressed as
$\mathrm{AF}=2 \sum_{n=1}^{N} I_{n} \cos \left[\left(\frac{2 n-1}{2}\right) k d \sin (\theta)+\varphi_{n}\right]$

Where $\theta$ denotes the zenith angle measured from the broadside direction of the array, $I_{n}$ is the current excitation amplitude and $\varphi_{n}$ (which is fixed at zero) is the phase of the $n$th array element, $d$ is the inter element spacing between the radiating elements, and the wave number of the carrier signal is $\mathrm{k}=2 \pi / \lambda$. Where $\mathrm{kd}$ is taken as $\pi$ (for $\mathrm{d}=\lambda / 2$ ).

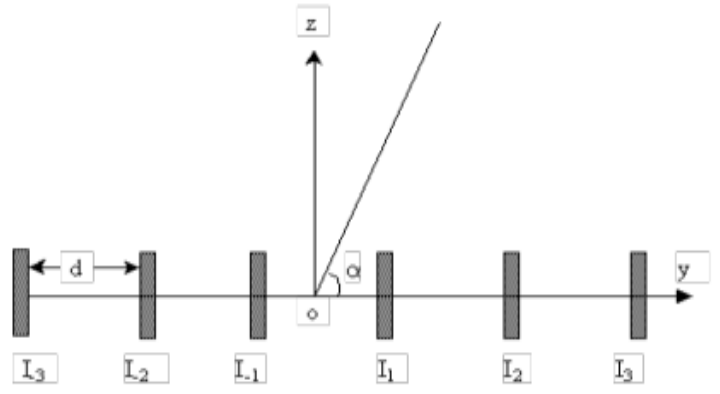

Figure 2: N-element linear array [5].

After defining the radiation pattern, next is to formulate the (objective) function be minimized. The fitness function is from the array factor in such that the objective of the optimization is satisfied. The general form of the fitness function is given by

$\mathrm{FF}=\sum_{\theta=-90^{0}}^{90^{0}} w(\theta)\left[F_{0}(\theta)-F_{d}(\theta)\right]$

Where $F_{0}(\theta)$ is the pattern obtained using the proposed algorithm and $F_{d}(\theta)$ is the desired pattern. Here it is taken to be the Chebychev pattern with SLL of $-13 \mathrm{~dB}$ and $w(\theta)$ is the weight vector to control the sidelobe level in the cost function.

\section{RESULTS}

The research work applied Dolph Chebyshev synthesis method to synthesized the antenna array for $\mathrm{N}=10,16,20,24$ with 00 scan angle and an inter-element spacing of $\lambda / 2$ as a reference. The amplitude distribution of Dolph Chebyshev technique gives a maximum side lobe level of $-35 \mathrm{~dB}$ for the entire antenna array used. RGA was executed with 500 iterations, and the population size of 12 . A uniform crossover was used with mutation probability of $4 \%$ and a maximum generation of 500. The program was written and ran with MATLAB (R2008b) on a system with $3.00 \mathrm{GHz}$ core (TM) 2 duo processor and a Memory of 4GB. Best results that gave the minimum side lobe level for the antenna pattern were found for each iteration and are shown in table 1.0. The radiation patterns for $\mathrm{N}=10,16,20,24$ in both case are also shown in Figure 3, 4, 5 and 6

Also it can be observe from Figure $3 \mathrm{a}$ through Figure $6 \mathrm{~b}$ that, as the current amplitude tapers from the center to the edges of the array increased, the side-lobe level decreased, but with an accompanying increase in the width of the main beam. Also as the number of antenna array increases, the main beamwidth decreases and the improvement obtained with RGA increases except with $\mathrm{N}=24$ where the improvement was little compared the others. 
Table 1: Current excitation weights and the SLL (dB) Obtained with RGA methods for various No of Array Elements

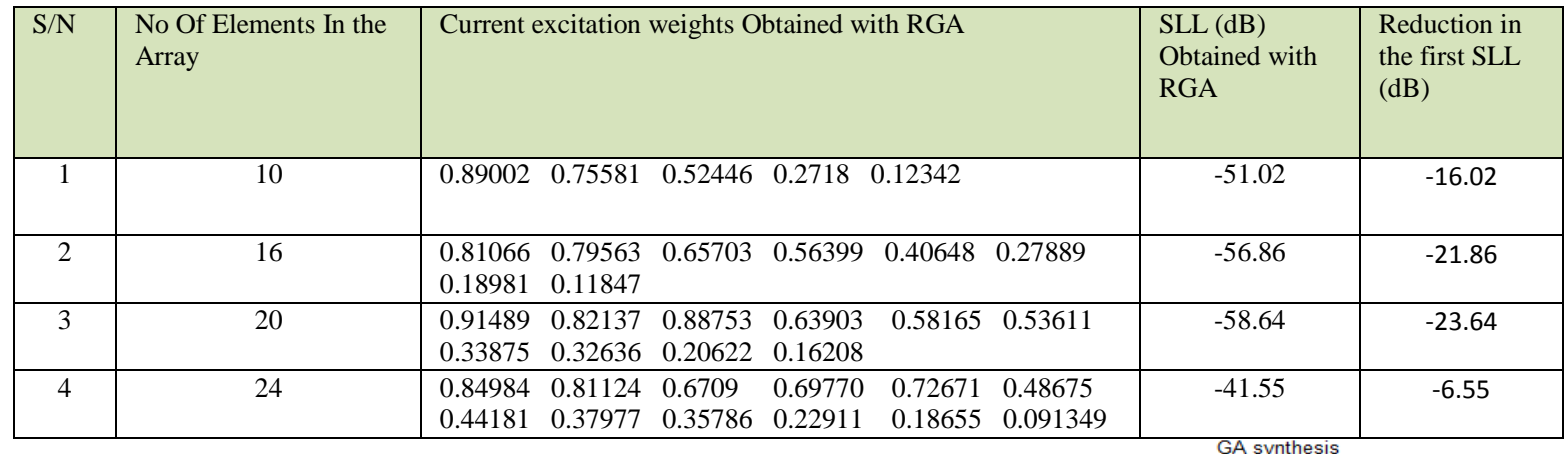

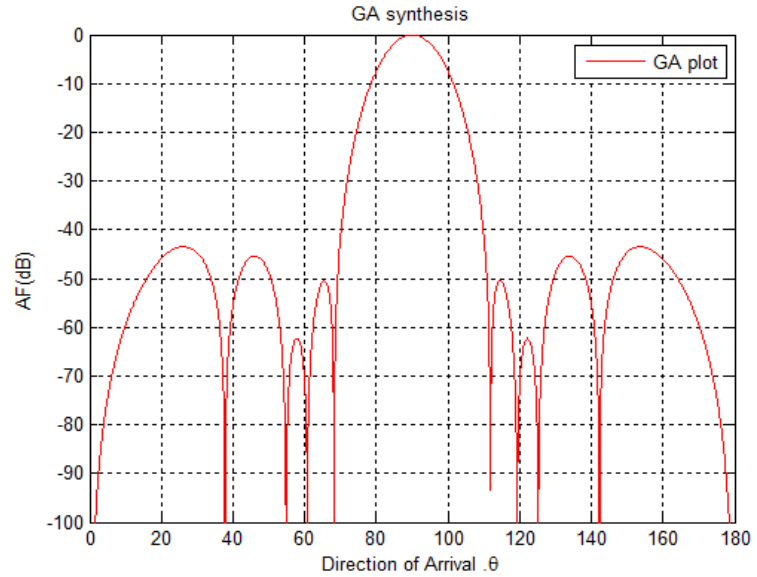

Fig 3a: Optimized Radiation pattern with reduced sidelobe level for $\mathbf{N = 1 0}$ elements array for GA method.

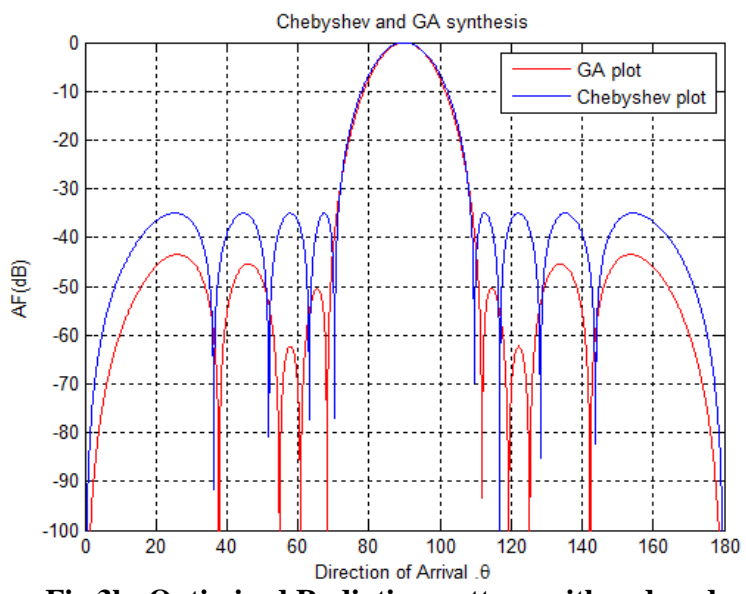

Fig 3b: Optimized Radiation pattern with reduced sidelobe level for $\mathbf{N}=10$ elements array for GA \& Dolph Chebyshev methods

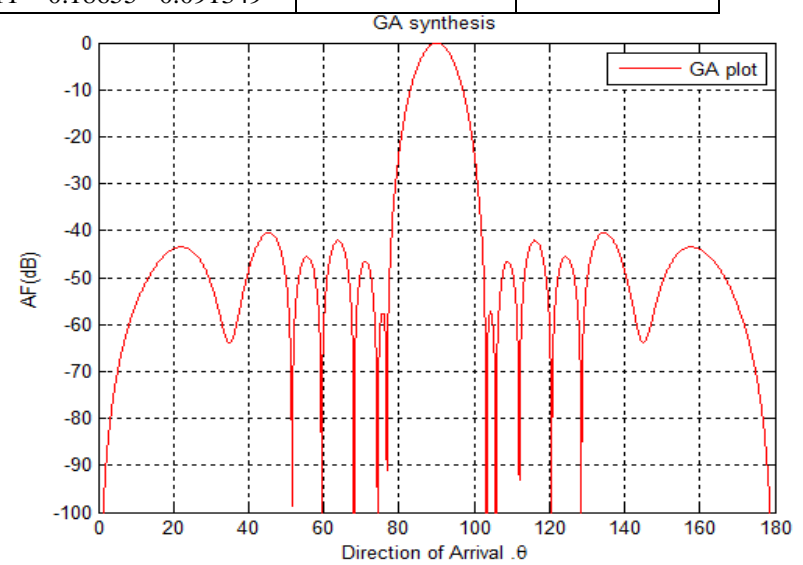

Fig 4a: Optimized Radiation pattern with reduced sidelobe level for $\mathbf{N}=16$ elements array for GA method

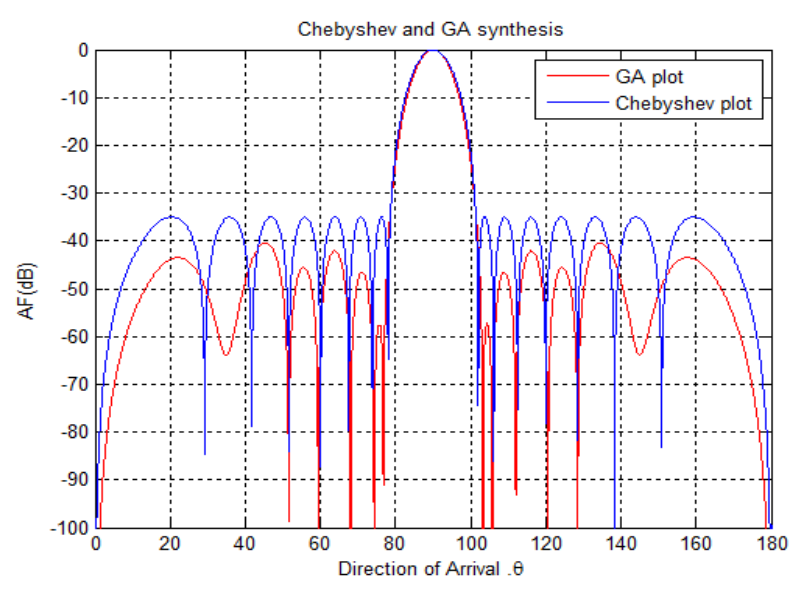

Fig 4b: Optimized Radiation pattern with reduced sidelobe level for $\mathbf{N}=16$ elements array for GA \& Dolph Chebyshev methods 


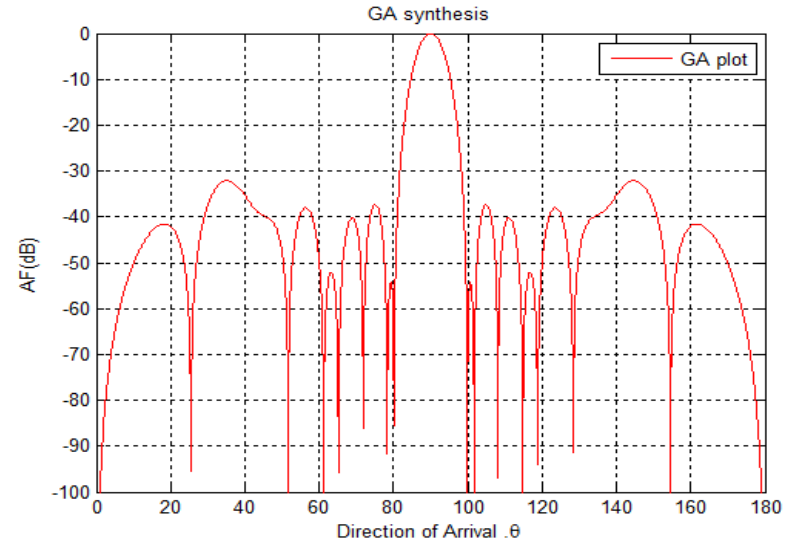

Fig 5a: Optimized Radiation pattern with reduced sidelobe level for $\mathbf{N}=\mathbf{2 0}$ elements array for GA method.

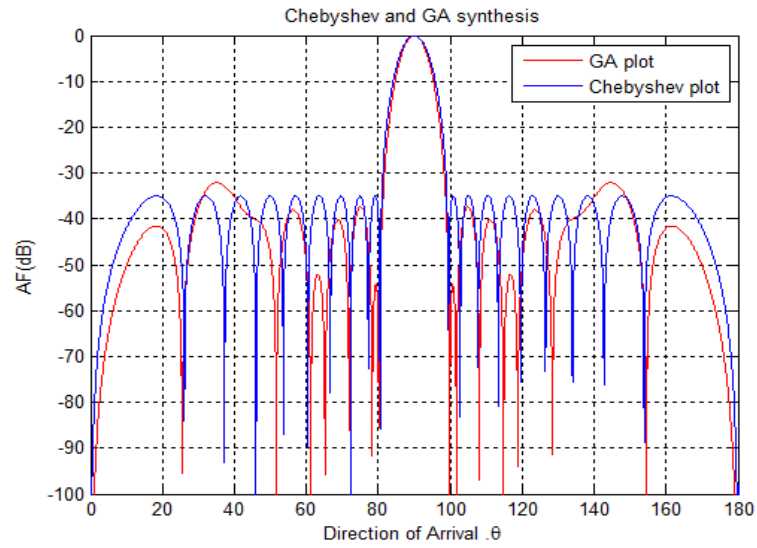

Fig 5b: Optimized Radiation pattern with reduced sidelobe level for $\mathbf{N}=20$ elements array for GA \& Dolph Chebyshev methods

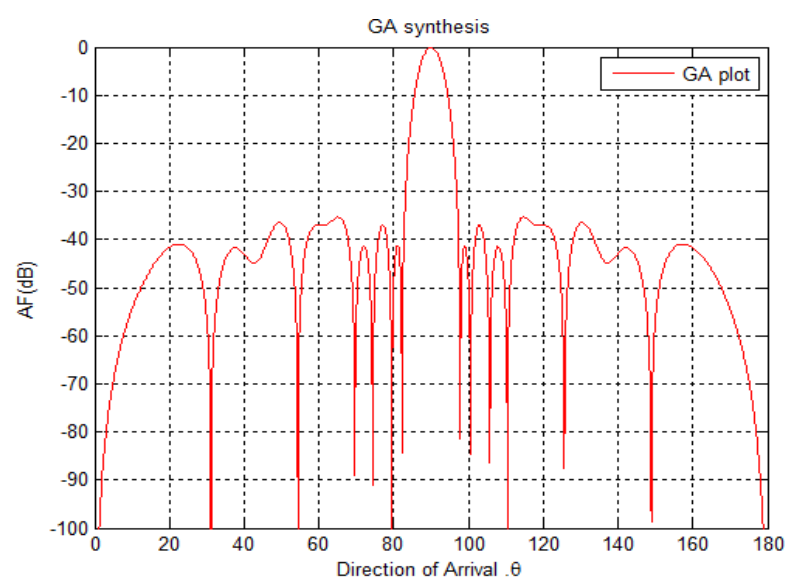

Fig 6a: Optimized Radiation pattern with reduced sidelobe level for $\mathrm{N}=\mathbf{2 4}$ elements array for GA method.

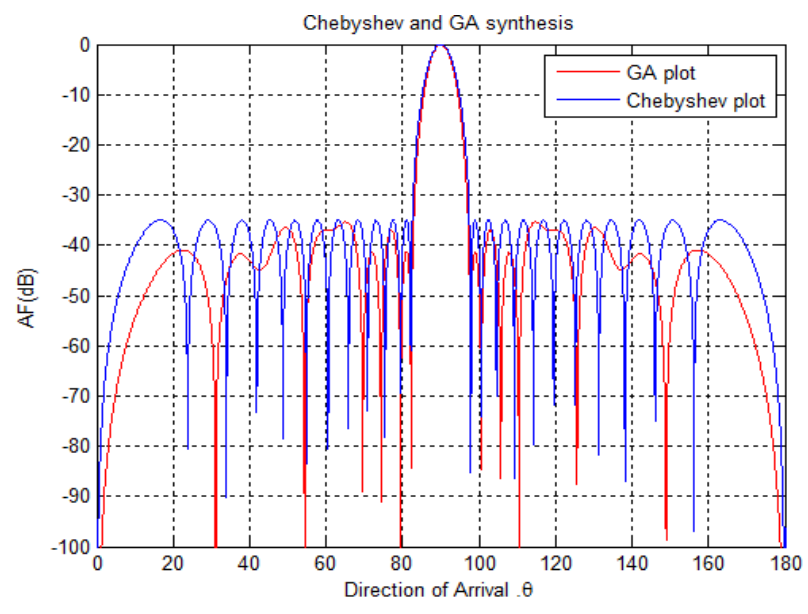

Fig 6b: Optimized Radiation pattern with reduced sidelobe level for $\mathrm{N}=\mathbf{2 4}$ elements array for GA \& Dolph Chebyshev methods

\section{CONCLUSION}

This research work proved the power of using Genetic algorithm in solving complex problems where you have conflicting constrains. The first side lobe level which introduces Electromagnetic Interference (EMI) problems is lowered without disturbing the beam width. RGA finds an optimum compromise between the beamwidth and side-lobe level with $\mathrm{N}=10,16,20$ and 24 . It can be seen that the current excitations obtained from RGA is lower than that of Dolph Chebyshev for all the number of elements simulated, while at the same time, the first null to null beamwidth is slightly reduced. The excitation levels of Dolph Chebyshev method are tapered in nature from the centre of the array. However, the excitation levels computed using RGA is random in amplitude with no regular taper. But the excitation levels in both the cases are symmetric to the centre of the array

\section{ACKNOWLEDGMENTS}

The authors would like to thank Engr. Hassan A. Basher and Dr. Dahiru S. Sani and Engr. Amir A. Bature for their valuable contributions towards the achievement of this research paper. Our appreciation also goes to authors whose works have been cited and duly referenced in this article.

\section{REFERENCES}

[1] S. Joseph, R. Kulandai, and S. Joerg, Matlab Modelling, Programming and Simulations. India: MathWorks, Inc., September 2010.

[2] T. Laseetha and R. Sukanesh, "Synthesis of linear antenna array using genetic algorithm with cost based roulette to maximize side lobe level reduction," WSEAS Transactions on Communications, vol. 10, pp. 385-394.

[3] A. K. Aboul-Seoud, A. K. Mahmoud, and A. E. S. Hafez, "A sidelobe level reduction (SLL) for planar array antennas withân $30 \mathrm{~dB}$ attenuators weight precision," Aerospace Science and Technology, vol. 14, pp. 316-320.

[4] P. Joshi and R. Dubey, "Optimization of linear antenna array using genetic algorithm for reduction in Side lobs levels and improving directivity based on modulating parameter M," Optimization, vol. 1.

[5] S. Shrivastava, "Broadside linear antenna array synthesis using Genetic Algorithm," International Journal of 
Scientific Research Engineering \& Technology (IJSRET), vol. 2, pp. 332-336, September 2013.

[6] K. Man, K. Tang, and S. Kwong, "Genetic algorithms: concepts and applications [in engineering design]," Industrial Electronics, IEEE Transactions on, vol. 43, pp. 519-534, 1996.

[7] G. A. Thiele, Antenna theory and design: Wiley, 1998.

[8] P. M. Mainkar, S. S. Ghule, O. S. Ghate, and R. N. Ojha, "Optimal Side Lobe Reduction Of Linear Non-Uniform Array Using Genetic Algorithm," International Journal of Computer Architecture and Mobility, vol. 1, April 2013.
[9] V. Rajya Lakshmi and G. Raju, "Pattern Synthesis using Genetic Algorithm for Low Sidelobe Levels," International Journal of Computer Applications, vol. 31, pp. 53-57.

[10] T. S. Jeyali Laseetha and R. Sukanesh, "Synthesis of Linear Antenna Array using Genetic Algorithm to Maximize Sidelobe Level Reduction," International Journal of Computer Applications, vol. 20, 2011.

[11] F. B. Gross, Smart antennas for wireless communications: with MATLAB: McGraw-Hill New York, 2005. 